\title{
3

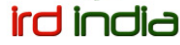

\section{Advancing of Industrial Field Using Embedded Web Server for Remote Monitoring and Controlling}

\author{
${ }^{1}$ K.Pothuluraiah, ${ }^{2}$ A.Suman Kumar \\ ${ }^{1}$ [M.Tech], ${ }^{2}$ Associate Professor, PBR Visvodaya Institute of Technology and Science, \\ Nellore, Andhra Pradesh, India.
}

\begin{abstract}
Embedded Ethernet is nothing but a microcontroller which is able to communicate with the network currently device with microcontroller has been widely used in industrial field. However, a large number of devices don't have the network interface and the data from them cannot be transmitted in network. A design of ARM Processor-based embedded Ethernet interface is presented. In the design, an existing SPI serial device can be converted a network interface peripheral to obtain compatibility with the network. By typing the IP-Address of LAN on the web browser, the user gets a web page on screen; this page contains all the information about the status of the devices. The user can also control the devices interfaced to the web server by pressing a button provided in the web page.
\end{abstract}

Index terms - Embedded Ethernet; ARM Processor; SPI; Web server

\section{INTRODUCTION}

Computer communication systems and especially the internet are playing an important role in the daily life. Using this knowledge many applications are imaginable. Home automation, utility meters, appliances, security systems, card readers, and building controls, which can be easily, controlled using either special front-end software or a standard internet browser client from anywhere around the world. Web access functionality is embedded in a device to enable low cost widely accessible and enhanced user interface functions for the device.

The remote and monitoring system can be control by many kind of different .technology. The progress can be control and monitor system via field bus, and Ethernet, power line carrier, SMS, EPRS, ADSL, GPRS, CDMABased, 3G-Based

Wireless network, telephone control and other communication means are applied to realize data transmission between data acquisition modules and data collection center. The remote and monitoring able system can be the temperature control and monitoring system, streetlight monitors and control system, remote monitoring of air-quality system, online power system, crop field remote monitoring system, remote measurement and control system for greenhouse, home automation system and some other system that will appear on our living life. Remote monitoring systems based on web-server-embedded technology and mobile telecommunication will become a core node technology in sensing network construction because of a great deal of mobile users and spreads of digital services in next generation telecommunication in the world. Soil, environmental, and crop information monitoring are important in production management and decision making in precision agriculture. Therefore, reliability, security and inexpensive characteristics required will be essential in the crop field information monitoring. Three improved field monitoring servers (FMS) using code division multiple access (CDMA) services combined with IPSec-based virtual private network (VPN) function have installed to two rice practical fields in Shanghai and one maize experimental field in Beijing for constructing a remote wireless sensing network. This crop field remote monitoring system as a ubiquitous node infrastructure in wireless sensing networks is useful and powerful to collect soil, environment, and crop information in remote for precision agriculture. The real-time soil and environment data, and crop images can be dynamically collected in remote area by the crop field monitoring systems in remote. This crop field remote monitoring system using web-server-embedded technology and CDMA service with IP Sec based VPN function as a node infrastructure is powerful and useful to construct ubiquitous wireless sensing network in lowcost and high-security for crop production .A web server in the devices provides access to the user interface functions for the device through a device web page. A web server can be embedded into any appliance and 
connected to the Internet so the appliance can be monitored and controller from remote places through the browser in a desktop. Temperature, pressure, displacement, motion and sound are the most often measured environmental quantities.

Among these environmental quantities, temperature is the most often measured parameter in industries. For example, some processes work only within a narrow range of temperatures; certain chemical reactions, biological processes, and even electronic circuits perform best within limited temperature ranges. So, it is necessary to measure the temperature and control if it exceeds some certain limit to avoid any misbehavior of the systems. To accurately control process temperature without extensive operator involvement, a temperature control system relies upon a controller, which accepts a temperature sensor.

An ARM processor based embedded Ethernet interface system is designed. In the system, the introduced microcontroller LPC2138 can communicate with serial data acquisition equipments at the terminal through SPI interface and can transmit data to remote host computer through Ethernet interface. Compared with the system a host is connected to many serial devices, the task of host is only to complete a single Ethernet communication and its load is lower.

\section{SYSTEM DESIGN}

Many embedded systems have substantially different designs according to their functions and utilities. In the design, structured modular design methods adopted and the system is mainly composed of SPI communication module, processor module and Ethernet interface module, as shown in Fig. 1. The figure shows that the typical architecture of an embedded Ethernet monitor and control using web browser architecture.

Here all the devices are connected to the processor and the ADC converters are used to convert analog data into digital data. Serial communication is done in between processor and Ethernet controller CS 8900A.It is connected to LAN cable through RJ 45 registered jack and the whole device is connected to remote $\mathrm{PC}$ through internet.

\section{- $\quad$ LPC2138 Processor}

LPC2138is a microcontroller which is based on a supporting embedded tracking 32bit ARM7TDMI-STM CPU and real-time simulation, with a high-speed Flash memory of $512 \mathrm{KBembedded,} \mathrm{128bit} \mathrm{width} \mathrm{of} \mathrm{memory}$ interface and unique accelerating structure; make the 32 bit code to run in the maximum clock rate. part features of lpc2138,two eight-way 10bits A/D converters contains 16analog inputs, each channel's conversion time is 2.44 microseconds. One $10 \mathrm{bit} \mathrm{D} / \mathrm{A}$ converter can provide different simulation outputs.

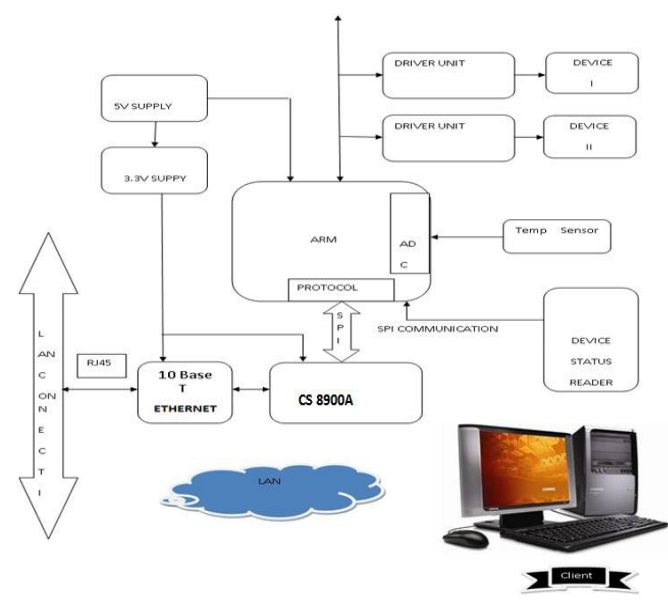

Fig 1: System Architecture

Two 32bit timer/counter (with 4 ways to capture and 4 comparison channels) and PWM unit (6 output roads) and watchdog. Real-time clock has independent power source and clock source, and in power saving mode, it dramatically reduced energy consumption. Several serial interface, include two $16 \mathrm{C} 550$ industrial standard UART, two high-speed I2C interface (400kbit/s) SPITM and SSPC (with buffer function, the data length variable). Vector interrupt controller. It can be configurable priority and vector address. As many as 47 general I/O ports of 5v LQFP64 encapsulation. The scope of crystal frequency within the piece $1 \sim 30 \mathrm{MHz}$. Two low power consumption patterns free and power down. The CPU operating voltage range $3.0 \sim 3.6 \mathrm{~V}$ $(3.3 \mathrm{v}+/-10 \%)$. I/O port can with stand the maximum voltage of $5 \mathrm{v}$. It is applied in industrial control, medical systems, POS machines, communication gateways, embedded software modern aspects. Ethernet Interface

Because of the ARM system without the Ethernet interface, its application value will be greatly discounted, so regard as the whole system, the Ethernet interface circuit is essential, but complicated. Form the view of hardware, as shown in figure 2, the ITU.T802.3 model layer structure, the Ethernet interface mainly contains MAC controller and physical interface.

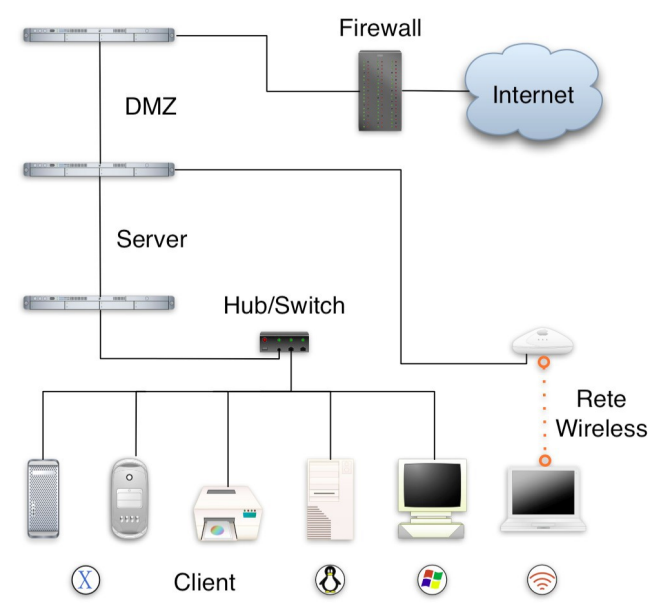

Fig 2: Ethernet Communication Module 
LPC2138 is embedded with MAC, but it doesn't provide physical interface .so it uses a DM9000A chip to provide Ethernet access channels. DM9000A integrates 10/100Mbps physical interface, with an 16Kbyte SRAM which is used to send and receive the FIFO cache, it supports two 8/16bit host modes, and it supports directly interconnect flipping function, and it supports accelerated TCP/IP and so it reduces the burden of CPU and improves the efficiency and the reading and writing $\mathrm{I} / \mathrm{O}$ time is $10 \mathrm{~ns}$ only. DM9000A Ethernet controller observes 802.3 Ethernet transfer protocol promulgated by IEEE. The actual design application connection is showed by figure 2 .

\section{- $\quad$ System Software Design}

In order to transmit the data from SPI serial to Ethernet, two system tasks are established in OS $\mu \mathrm{C} / \mathrm{OS}-\Pi$. One is to receive front-end data through SPI interface and the other is to transmit data to Ethernet.

\section{- $\quad$ SPI Receiving task:}

For the case that the data are transmit to Ethernet, the data which have been arrived at SPI port are stored into SPI sending buffer and packaged according to TCP/IP protocol and then are added IP and UDP message head on the condition that the PC with SPI interface is set to SPI slave mode and the SPI interface is enabled. At last, the converted data are sent to the host through the corresponding UDP port. This process is shown in Fig. 3(a).

\section{- $\quad$ Ethernet Receiving task:}

In the Ethernet task, in order to receive the data from Ethernet in the system, the local IP address and subnet mask must be set firstly, and the appropriate UDP port is opened to monitor whether there are data in UDP port.

As UDP packet, the data which have been reached the UDP port, are analyzed according to TCP/IP protocol and then stored into SPI receiving buffer. At last, the analyzed data are sent to the SPI serial device through SPI interface driver. In the process above, an infinite loop is used and the process is shown in Fig. 3(b).

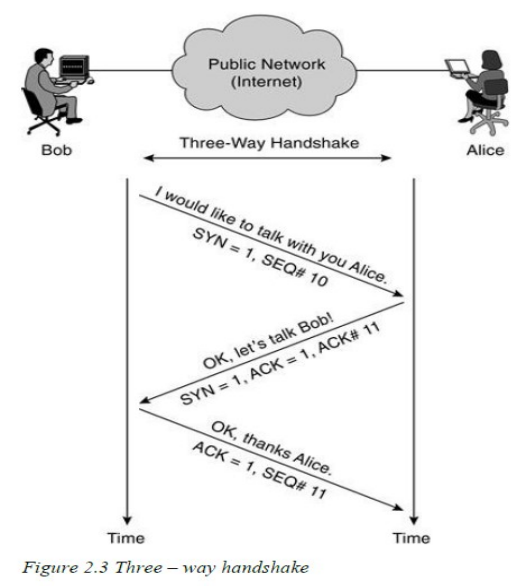

Fig 3: Tasks for transmitting Data

\section{SYSTEM TESTING}

Embedded Ethernet circuit board is shown in the fig (4).The circuit board consist of ARM processor, Ethernet interface module and rj45 registered jack. The devices which are to be controlled are connected to the circuit board. The snap shots for controlling the AC motor and monitoring the temperature sensor is given below.

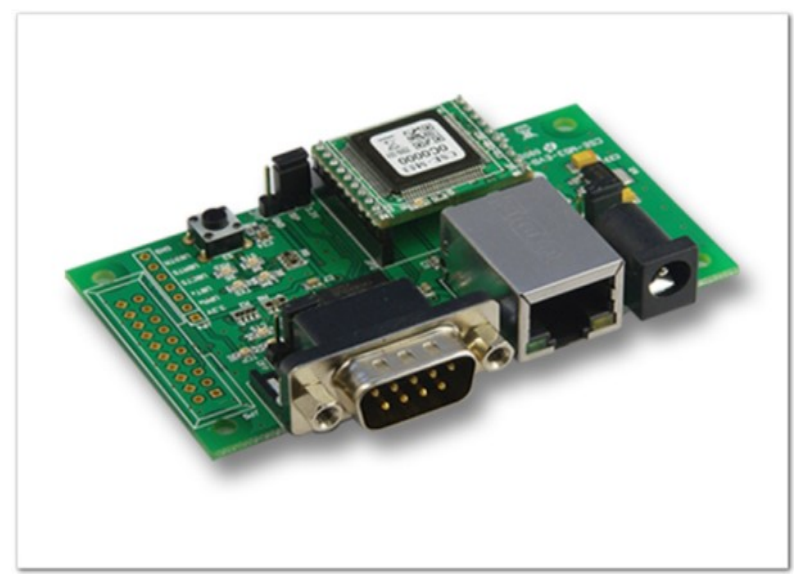

Fig 4: Embedded Ether net Circuit Broad

Fig. (5) \& (6) shows the simple web page designed using HTML language. It is requested by the client to server. Then the internet processes these request and server response for client request with web page. Now the Client can know the status of industry machineries and can control the machines via its own browser from remote location.

The status of the AC motor is shown on the web page by typing the IP address of the server board. We can view the status of AC motor as shown and change the status by clicking on it and update the system. The status of the AC motor is changed similarly the temperature of the room will be observed for every hour by refreshing the results. Hence, results show that the client can access the whole industry from any remote place via its own local browser. In industry the single ARM board acts as data acquisition and control system and as web server, so the system is compact with less complexity.

\section{IV.CONCLUSION}

In order to transmit the data from an existing device with SPI interface to network, an embedded Ethernet monitor and controlling system based on web browser is designed. This design can be used widely in remote data acquisition and control system in industry. 


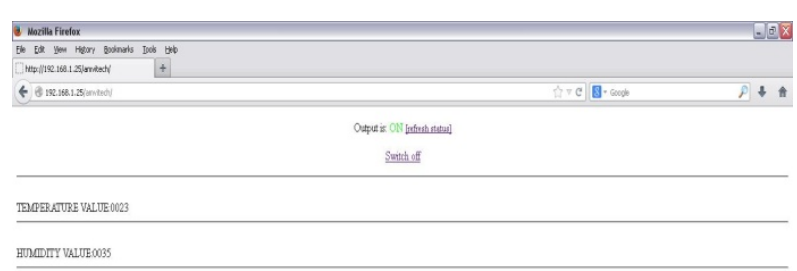

Fig 5: Web page showing the status and Control of AC motor

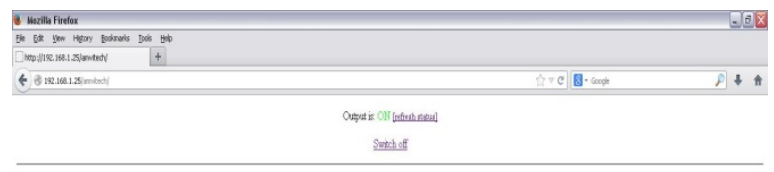

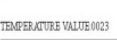

Enerrryursouss

Fig 6: web page showing the status of temperature sensor and Humidity

These Embedded Ethernet modules are having the capacity to perform as a true Ethernet device. It is possible to interface different kind of sensors with these modules and make various applications. So it can monitor embedded system operation state through Internet, achieving network monitoring purposes. The ARM system adopts the high performance Ethernet controller, the system communication and debugging are fast, reliable and real-time; In addition, it can be also applied in on-line monitoring, remote fault diagnosis system.

\section{REFERENCES}

[1] Arul Prabahar A, Brahmanandha Prabhu, "Development of a Distributed Data Collection System based on Embedded Ethernet" 978-14244-9799-71111\$26.00 (C2011 IEEE.

[2] Hua Fu, Tao Wang, Cui Yang. Gas monitoring system based on ARM and information fusion $[\mathrm{J}]$. Application of electronic technique. 2009(11) 39-42.

[3] CHEN Guo-ju, "Design of a monitoring system based on ARM and Etherne applied to AC motors," Journal of Nanjing Institute of Technology (Natural Science Edition), Magn. China, vol.7 (2), pp.46- 51, Jun. 2009.

[4] YU Cheng-bo, LIU Jie, and TAO Hong-yan, "Reseach on remote monitor technology of equipment," Information and Control, Magn. China, vol.31 (3), pp.236-240, June 2002.

5] Tao Lin ,Hai Zhao ,Jiyong Wang ,Guangjie Han and Jindong Wang,"An Embedded Web Server for Equipment ",School of Information Science \& Engineering, Northeastern University, Shenyang, Liaoning, China.

B. Rajesh Kumar, Member, IEEE, K. Sridharan, Senior Member, IEEE, and K. Srinivasan,"The Design and Development of a Web-Based Data Acquisition System", ieee transactions on instrumentation and measurement, vol. 51, no. 3, june 2002

[7] Zhao Hai, "Embedded Internet - an information technology revolution of 21 st century," Beijing: Tsinghua University Press, 2002, pp. 198-225

[8] Ximin Zhang, Junding Sun, Lihua Zhou, "Development Of an Internet Home Automation System using Java and Dynamic DNS Service", Sixth International Conference on Parallel and Distributed Computing, Applications and Technologies, 2005.

[9] Atmel Corporation. Embedded web server. A VR 460, January 20 I 0. 